\title{
Vibrational echo spectral observables and frequency fluctuations of hydration shell water around a fluoride ion from first principles simulations $^{\dagger}$
}

\author{
DEEPAK OJHA and AMALENDU CHANDRA* \\ Department of Chemistry, Indian Institute of Technology Kanpur, Kanpur, Uttar Pradesh 208 016, India \\ E-mail: amalen@iitk.ac.in
}

MS received 21 February 2017; accepted 20 March 2017

\begin{abstract}
Aqueous solution of a fluoride ion at $300 \mathrm{~K}$ is studied using the method of $a b$ initio molecular dynamics simulation. Instantaneous fluctuations in vibrational frequencies of local OD stretch modes of deuterated water are calculated using a time-series analysis of the simulated trajectory. The vibrational spectral diffusion of OD modes in the first and second solvation shells and also in bulk of the aqueous fluoride ionic solution are studied through calculations of the frequency time correlation function (FTCF), joint probability distributions, slope of three pulse photon echo (S3PE) and two dimensional infrared spectrum (2D-IR). The vibrational spectral dynamics in the first solvation shell shows decay with three components which can be correlated with the dynamics of intact ion-water hydrogen bonds, ion-water hydrogen bond lifetime and the escape dynamics of water molecules from the solvation shell. The vibrational spectral diffusion of OD modes in the second solvation shell and in the bulk show very similar decay behavior. The timescales obtained from FTCF, S3PE and the slope of nodal line (SNL) of 2D-IR are found to be in reasonable agreement with each others.
\end{abstract}

Keywords. Vibrational echo; frequency fluctuations; hydration shell water; fluoride ion; ab initio molecular dynamics.

\section{Introduction}

Ions dissolved in liquid water play important roles in several chemical and biological processes. ${ }^{1,2}$ Similarly, water molecules in aqueous ionic solutions exhibit different dynamics in comparison to pure water. ${ }^{2-4}$ There are several aqueous phase chemical reactions like $\mathrm{S}_{N} 2$ nucleophilic substitution, unimolecular dissociation and acid dissociation reactions which involve solvated anions. ${ }^{5}$ In view of their huge importance in both energetics and dynamics of aqueous phase chemical reactions, aqueous ionic solutions have been studied extensively using both experimental and theoretical methods. ${ }^{2-15}$ The arrangement of water molecules around fluoride ions has been studied over a range of concentration in potassium fluoride solutions using the neutron diffraction technique. ${ }^{16}$ Recently, with the advent of time-dependent vibrational spectroscopy, there have been several spectroscopic studies which have intensively focused on solvation shell dynamics

\footnotetext{
*For correspondence

${ }^{\dagger}$ Dedicated to the memory of the late Professor Charusita Chakravarty.
}

and rate of vibrational spectral diffusion in aqueous solutions. In this regard, we note in particular the pumpprobe experiments of aqueous ionic solutions which could differentiate the dynamics of water molecules in the solvation shell from those present in the bulk. Early experiments in this area found the decay of frequency correlations of water in the hydration shells of halide ions to be in the range of 10-25 ps. ${ }^{6}$ Subsequent experiments using improved techniques reported vibrational spectral diffusion timescale of $4.7 \mathrm{ps}$ for $6 \mathrm{~m} \mathrm{NaBr}$ solution. ${ }^{17}$ The orientational dynamics of water molecules in ionic solutions has also been studied through femtosecond pump-probe spectroscopy. It was found that the rotational dynamics of water molecules beyond the first solvation shell was not influenced by the presence of ions. ${ }^{7}$ Similarly, there has also been a study of equilibrium dynamics of thiocyanate chemical exchange between free thiocyanate ion and calcium or magnesium thiocyanate complex using two dimensional infrared (2D-IR) spectroscopy. ${ }^{5}$

On the theoretical side, the structural order of water molecules around fluoride ions has been studied through classical molecular dynamics and combined quantumclassical (QM/MM) technique. ${ }^{18,19}$ Dynamic evolution 
of the solvation shells around ions was found to be slower in these studies. The effects of temperature and pressure on the hydration of ions in water and also the rotational motion of water molecules in the solvation shells have also been reported. ${ }^{20} \mathrm{~A}$ recent study ${ }^{21}$ reported calculations of the pump-probe spectrum of aqueous solution of iodide ions using classical simulations. The long-time relaxation of frequency fluctuations was shown to be related to the escape dynamics of water from the solvation shells of iodide ions. ${ }^{21}$ The frequency fluctuations in solvation shells of halide ions have also been studied using ab initio molecular dynamics without involving any empirical models. ${ }^{22}$ These studies also calculated the average residence time of water in the hydration shells of halides using population time correlation approach and found an average residence time of 20-30 ps for these anions. Further, studies have also been reported for calculationis of the transient hole burning spectroscopy and 2D-IR spectroscopy of water and aqueous ionic solutions from first principles simulations. ${ }^{22-24}$

In the present study, we present calculations of the non-linear vibrational spectroscopy of water molecules in the solvation shell of a fluoride ion and of bulk water from first principles simulations. We have performed the spectral calculations within the framework of second order cumulant approximation. We note that although the second order cumulant approximation for liquids with complex hydrogen bond network like water is not completely valid due to non-Gaussian features, ${ }^{25-30}$ the spectral diffusion timescales calculated within the approximation are found to be in reasonable agreement with the timescale of frequency correlation for water and ionic solutions. ${ }^{23}$ In the present study, we have calculated the 2D-IR spectrum, slope of 3-pulse photon echo (S3PE) (an analogue of the peak shift) and frequency time correlation function (FTCF) of water in the first solvation shell, second solvation shell and also in the bulk. We have obtained the timescales of spectral diffusion from FTCF, normalized S3PE and slope of nodal line (SNL) of the calculated 2D-IR spectrum. We have calculated the fluctuations in vibrational frequencies of OD modes using the wavelet method of time-series analysis. In order to determine the fluctuations in the excited state, we have modeled the OD modes as Morse oscillators. The method has been described in details in our previous work. ${ }^{23}$ We note that most of the experimental and simulation studies have looked at the spectral features of HOD in ionic solution of $\mathrm{H}_{2} \mathrm{O}$ or $\mathrm{D}_{2} \mathrm{O} .{ }^{31-39}$ In the present study, we have considered an ionic solution of fluoride ion in $\mathrm{D}_{2} \mathrm{O}$. The spectral dynamics of an isolated $\mathrm{OD} / \mathrm{OH}$ mode of HOD in ionic solution from $a b$ initio perspective remains a challenging task within the existing computational framework.

\section{Theoretical formulation}

In the current section, we present the theoretical formulation for the calculation of the response functions of the third order polarization as given by Mukamel. ${ }^{40-42}$ When a system is targeted with three electric field pulses with the propagation vectors $k_{1}, k_{2}$ and $k_{3}$, a macroscopic polarization is produced in the rephasing direction which is often termed as an echo and also a free induction decay (FID) in the non-rephasing direction. While the detailed derivation is available in the literature, ${ }^{40,41}$ we present here only the equations for response functions within the second order cumulant approximation. In the present study, we ignore the excited state lifetime effects and rotational effects. We refer to the time delay between the first and second pulses as $t_{1}$, the time delay between the second and third pulses as $t_{2}$ and the time after the third pulse onward as $t_{3}$. Within the above mentioned framework, the response functions are given as, ${ }^{40}$

$$
\begin{aligned}
R_{1}\left(t_{3}, t_{2}, t_{1}\right)= & R_{2}\left(t_{3}, t_{2}, t_{1}\right) \\
= & \mu_{10}^{4} \exp \left(-i\left\langle\omega_{10}\right\rangle t_{3}+i\left\langle\omega_{10}\right\rangle t_{1}\right) \\
& \times \exp \left(-g_{1}\left(t_{1}\right)+g_{1}\left(t_{2}\right)-g_{1}\left(t_{3}\right)\right. \\
& -g_{1}\left(t_{2}+t_{1}\right)-g_{1}\left(t_{2}+t_{3}\right) \\
& \left.+g_{1}\left(t_{1}+t_{2}+t_{3}\right)\right), \\
R_{3}\left(t_{3}, t_{2}, t_{1}\right)= & -\mu_{10}^{4} \kappa^{2} \exp \left(i \Delta t_{3}\right) \\
& \times \exp \left(-i\left\langle\omega_{21}\right\rangle t_{3}+i\left\langle\omega_{10}\right\rangle t_{1}\right) \\
& \times \exp \left(-g_{1}\left(t_{1}\right)+g_{2}\left(t_{2}\right)-g_{3}\left(t_{3}\right)\right. \\
& -g_{2}\left(t_{2}+t_{1}\right)-g_{2}\left(t_{2}+t_{3}\right) \\
& \left.+g_{2}\left(t_{1}+t_{2}+t_{3}\right)\right), \\
R_{4}\left(t_{3}, t_{2}, t_{1}\right)= & R_{5}\left(t_{3}, t_{2}, t_{1}\right) \\
= & \mu_{10}^{4} \exp \left(-i\left\langle\omega_{10}\right\rangle t_{3}-i\left\langle\omega_{10}\right\rangle t_{1}\right) \\
& \times \exp \left(-g_{1}\left(t_{1}\right)-g_{1}\left(t_{2}\right)-g_{1}\left(t_{3}\right)\right. \\
& +g_{1}\left(t_{2}+t_{1}\right)+g_{1}\left(t_{2}+t_{3}\right) \\
& \left.-g_{1}\left(t_{1}+t_{2}+t_{3}\right)\right), \\
R_{6}\left(t_{3}, t_{2}, t_{1}\right)= & -\mu_{10}^{4} \kappa^{2} \exp \left(i \Delta t_{3}\right) \\
& \times \exp \left(-i\left\langle\omega_{21}\right\rangle t_{3}-i\left\langle\omega_{10}\right\rangle t_{1}\right) \\
& \times \exp \left(-g_{1}\left(t_{1}\right)-g_{2}\left(t_{2}\right)-g_{3}\left(t_{3}\right)\right. \\
& +g_{2}\left(t_{2}+t_{1}\right)+g_{2}\left(t_{2}+t_{3}\right) \\
& \left.-g_{2}\left(t_{1}+t_{2}+t_{3}\right)\right), \\
&
\end{aligned}
$$

where $g_{n}(t)(n=1-3)$ is related to the time correlations of fluctuating transition frequencies and the expressions for $g_{1}(t), g_{2}(t)$ and $g_{3}(t)$ are given by

$$
\begin{aligned}
& g_{1}(t)=\int_{0}^{t} d \tau^{\prime} \int_{0}^{\tau^{\prime}} d \tau^{\prime \prime}\left\langle\delta \omega_{10}(0) \delta \omega_{10}\left(\tau^{\prime \prime}\right)\right\rangle, \\
& g_{2}(t)=\int_{0}^{t} d \tau^{\prime} \int_{0}^{\tau^{\prime}} d \tau^{\prime \prime}\left\langle\delta \omega_{21}(0) \delta \omega_{10}\left(\tau^{\prime \prime}\right)\right\rangle,
\end{aligned}
$$


and

$$
g_{3}(t)=\int_{0}^{t} d \tau^{\prime} \int_{0}^{\tau^{\prime}} d \tau^{\prime \prime}\left\langle\delta \omega_{21}(0) \delta \omega_{21}\left(\tau^{\prime \prime}\right)\right\rangle .
$$

In Eqs. 2 and $4, \Delta=\left\langle\omega_{21}\right\rangle-\left\langle\omega_{10}\right\rangle$ and $\kappa$ is equal to $\mu_{21} / \mu_{10}$. It may be noted that $\Delta$ is zero for a harmonic oscillator, hence its non-zero value measures the extent of anharmonicity of the system. In a conventional 2D-IR spectroscopic experiment, the third order polarization is obtained as the convolution of the third order response function and the externally applied electric field pulses. In the present study, we assume the external electric fields to be delta functions. Further, we obtain the spectrum in frequency domain by Fourier transformation along two of the frequency axes as a function of one of the time interval which is taken to be the interval between the second and third pulses. The equations for the 2D-IR spectrum in the frequency domain as Fourier transforms of the response functions is expressed as, ${ }^{40-43}$

$$
S\left(\omega_{3}, t_{2}, \omega_{1}\right)=\operatorname{Re}\left[\sum_{i=1}^{6} S_{i}\left(\omega_{3}, t_{2}, \omega_{1}\right)\right],
$$

where

$$
\begin{aligned}
S_{i}\left(\omega_{1}, t_{2}, \omega_{3}\right)= & \int_{0}^{\infty} d t_{1} \int_{0}^{\infty} d t_{3} \exp \left(i \omega_{3} t_{3}\right. \\
& \left.-i \omega_{1} t_{1}\right) R_{i}\left(t_{1}, t_{2}, t_{3}\right),
\end{aligned}
$$

for $\mathrm{i}=1$ to 3 and

$$
\begin{aligned}
S_{i}\left(\omega_{1}, t_{2}, \omega_{3}\right)= & \int_{0}^{\infty} d t_{1} \int_{0}^{\infty} d t_{3} \exp \left(i \omega_{3} t_{3}\right. \\
& \left.+i \omega_{1} t_{1}\right) R_{i}\left(t_{1}, t_{2}, t_{3}\right),
\end{aligned}
$$

for $\mathrm{i}=4$ to 6 , respectively.

Similarly, in integrated three pulse photon echo peak shift (3PEPS) experiments, the integrated intensity along the rephasing direction is found out. The integrated echo intensity is expressed mathematically as,

$$
I\left(t_{1}, t_{2}\right)=\int_{0}^{\infty} d t_{3}\left|\sum_{i=1}^{3} R_{i}\left(t_{1}, t_{2}, t_{3}\right)\right|^{2} .
$$

The peak shift $t_{1}^{*}\left(t_{2}\right)$ for a given $t_{2}$ is defined as the value of $t_{1}$ for which the echo intensity exhibits a maximum. ${ }^{44,45}$ Theoretically, several alternatives to the peak shift (3PEPS) have been proposed. ${ }^{46}$ The slope of 3-pulse photon echo (S3PE) is also known to capture the frequency correlation loss at both short and long timescales. The slope of three pulse photon echo (S3PE) is determined from the initial slope of the integrated three pulse photon echo intensity. The expression for the calculation of $\mathrm{S} 3 \mathrm{PE}$ is given as,

$$
S\left(t_{2}\right)=\left.\frac{\partial I\left(t_{1}, t_{2}\right)}{\partial t_{1}}\right|_{t_{1}=0} .
$$

The normalized frequency time correlation function is then given by ${ }^{45-47}$

$$
C\left(t_{2}\right)=\frac{S\left(t_{2}\right)}{S(0)}
$$

\section{Ab initio simulations}

The ab initio molecular dynamics simulation of the present study has been performed using the method of Car-Parrinello ${ }^{48,49}$ and the CPMD code. ${ }^{50}$ The simulation system consists of a single fluoride ion and $107 \mathrm{D}_{2} \mathrm{O}$ molecules in a cubic box of edge length $15.0 \AA$. The edge length was determined from the known experimental density of the solution at $300 \mathrm{~K} .^{51}$ The electronic structure of the system was determined within the Kohn-Sham formulation ${ }^{52}$ of density functional theory. ${ }^{53}$ The inner electrons were treated using the norm-conserving pseudopotentials of TroullierMartins. ${ }^{54}$ The plane wave expansion of the inner electrons was truncated at the kinetic energy cut-off of 80 Ry. A fictitious mass of 800 au was assigned to the electronic orbital degrees of freedom and the coupled equations of motion were integrated using a timestep of 5 au.

Several recent studies have shown that the equilibrium structure, dynamics and phase diagram of water improves on incorporation of dispersion corrections. ${ }^{55-59}$ In view of this, in the present study we have used the BYLP functional with dispersion corrections. The dispersion corrections were incorporated using the D2 scheme of Grimme. ${ }^{60,61}$ The initial configuration of system was obtained from classical molecular dynamics simulation followed by equilibration in the NVT ensemble using $a b$ initio simulation for 15 ps. Finally, the production run was carried out for the duration of another $50 \mathrm{ps}$ in the microcanonical ensemble for calculations of the vibrational frequency fluctuations, various observables of vibrational echo and 2D-IR spectroscopy and other dynamical quantities. As mentioned earlier, we have employed the second order cumulant approximation for spectral calculations. Thus, it is assumed that the dynamics is Gaussian and the transition dipole moment is independent of nuclear degrees of freedom. The ratio of the $0-1$ and 1-2 transition dipole moments $(\kappa)$ was determined using the eigenfunctions of Morse oscillators.

\section{Results and Discussions}

\subsection{Frequency fluctuation dynamics}

The time dependent fluctuations in the vibrational frequencies of OD modes in their ground states were calculated using a time-series analysis of the trajectory obtained from simulations. Further, the fluctuations in the higher excited states are obtained by modeling the OD modes as Morse oscillators. The details of both the 
methods are available in the literature. ${ }^{22,62,63}$ In order to identify the effects of the anion on vibrational dynamics of OD modes in different solvation shells, we have calculated the frequency distribution of OD modes present in the first and second solvation shells and also in the bulk which are shown in Figure 1. We have determined the solvation shells based on distance criteria. The OD modes found at a distance less than the first peak of the F-D radial distribution function from the fluoride ion, i.e., $2.5 \AA$, at a given time constitute the first solvation shell at that point of time. Similarly, OD modes which are found between $2.5 \AA$ and $4.5 \AA$ from the ion belong to the second solvation shell and remaining to the bulk. The average frequency of the OD modes present in bulk is found to be $2362 \mathrm{~cm}^{-1}$. Similarly, the average frequency distribution of OD modes present in the second solvation shell is also found to be 2362 $\mathrm{cm}^{-1}$ while that of OD modes present in first solvation shell is $2310 \mathrm{~cm}^{-1}$. We note that while the time averaged frequency distribution of OD modes present in the second solvation shell is essentially indistinguishable from that of the bulk, a significant red shift is seen for distribution of OD modes present in the first solvation shell. This implies that the OD modes present in the first solvation shell of the fluoride ion have stronger hydrogen bonds due to which the average distribution gets red shifted. This also implies that the effects of fluoride ion solvation over vibrational fre-

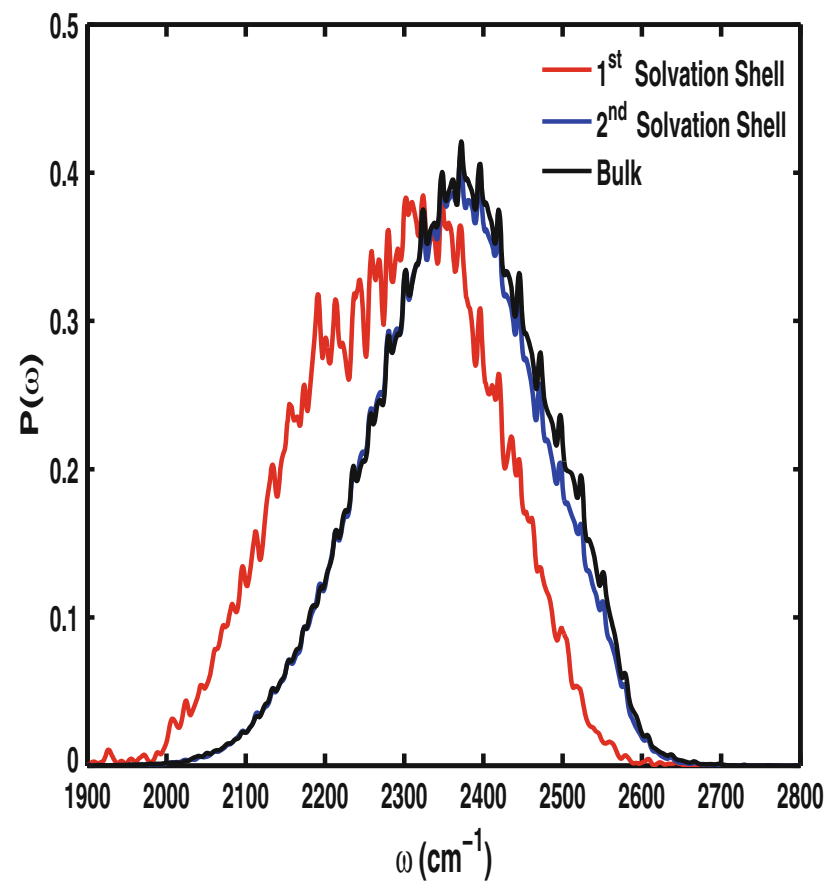

Figure 1. Frequency distributions of the OD oscillators in the first and second solvation shells and in the bulk regions of an aqueous solution containing a fluoride ion at $300 \mathrm{~K}$. quencies do not extend beyond the first solvation shell of the ion.

Further, we also calculated the frequency time correlation function (FTCF) of the OD modes in the first and second solvation shells and also in the bulk. The time dependent decay of FTCF of OD modes in different solvation shells is shown in Figure 2. We note that the decay of frequency correlation of bulk and second solvation shells are rather similar to each other. The timescales of decay of the frequency correlations of OD modes in the bulk and second solvation shell were determined by using a biexponential fit of the type shown below,

$$
f(t)=a_{0} \exp \left(-\frac{t}{\tau_{0}}\right)+\left(1-a_{0}\right) \exp \left(-\frac{t}{\tau_{1}}\right) .
$$

We note that the two timescale of decay of OD modes in the bulk are found to be $69 \mathrm{fs}$ and $2.54 \mathrm{ps}$. For the second solvation shell, the shorter timescale of decay is 75 fs while the component corresponding to the longer time decay is found to be $2.7 \mathrm{ps}$. It can be seen that the vibrational frequency relaxation dynamics of the second solvation shell and bulk are very similar. The timescales of decay of the frequency correlation of OD modes in the first solvation shell are found out by using a triexponential fit as given below,

$$
\begin{aligned}
f(t)= & a_{0} \exp \left(-\frac{t}{\tau_{0}}\right)+a_{1} \exp \left(-\frac{t}{\tau_{1}}\right) \\
& +\left(1-a_{0}-a_{1}\right) \exp \left(-\frac{t}{\tau_{2}}\right) .
\end{aligned}
$$

The short timescale of the decay is found to be around 50 fs while intermediate timescale is calculated to be $8.46 \mathrm{ps}$. The third longer timescale of the decay is found to be $27.5 \mathrm{ps}$. We note that the very fast timescale of the decay in the sub-femtosecond domain basically arises from the librational motion. Further, the intermediate time decay can be correlated with ion-water hydrogen bond lifetime and the very long third timescale of about $30 \mathrm{ps}$ can be attributed to the average residence time of water molecules in the ion solvation shell. The oscillations in decay of frequency correlation for the first solvation shell is mainly due to fluctuations in the interactions between the ion and water molecules present in the solvation shell.

We have also calculated the time dependent joint probability distributions of vibrational frequencies of OD modes that are present in the solvation shells and bulk regions. In the calculation of joint probability distribution for first solvation shell, the water molecules within the distance cutoff of less than the first minima of $F-O$ radial distribution function were considered. The results of these distributions are shown in 


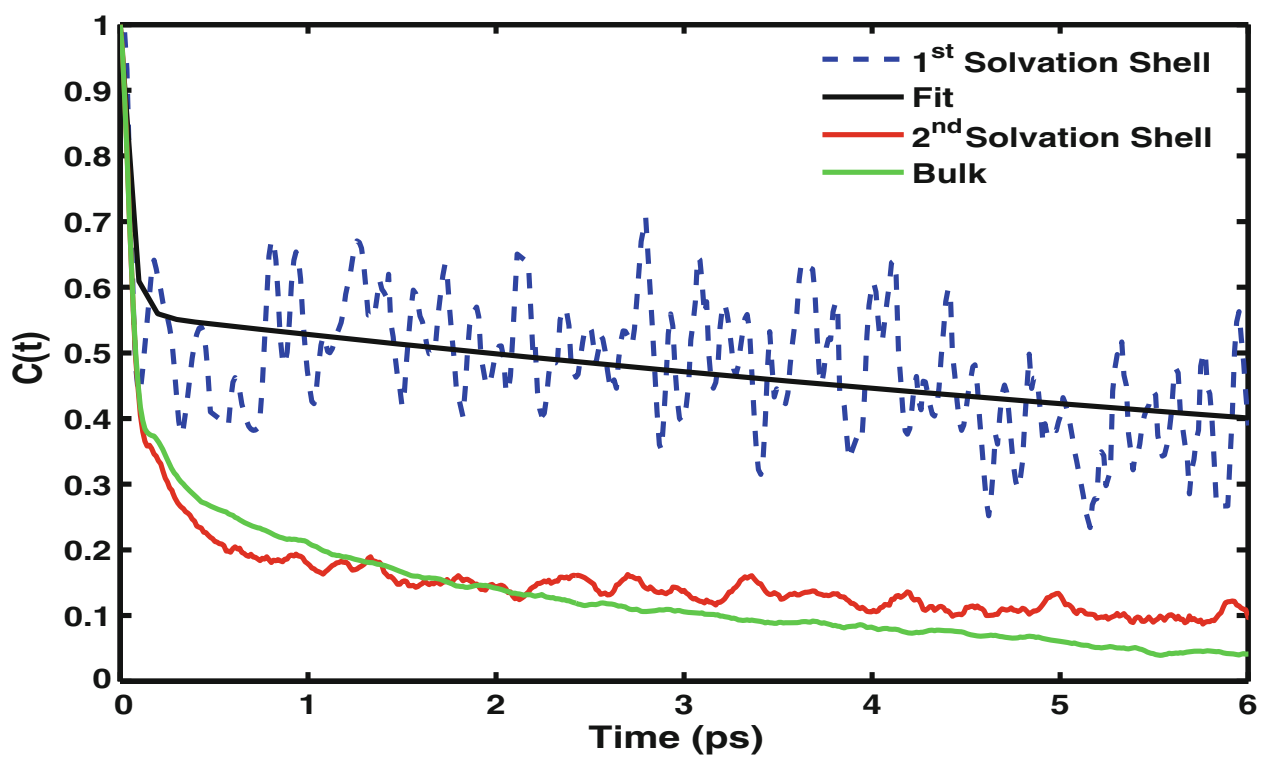

Figure 2. Time dependence of the correlation function of frequency fluctuations of OD stretch modes present in the first and second solvation shells and in the bulk of an aqueous solution containing a fluoride ion at $300 \mathrm{~K}$.

Figure 3. The joint probability distributions of OD modes for smaller waiting times, say of $10 \mathrm{fs}$, are found to be elongated along the diagonals. However, as the waiting times are increased to 3 ps, the distributions have become completely spherical for OD modes in the second solvation shell and bulk regions which implies loss of frequency correlations in such timescales. We note that the timescales obtained from the FTCF are also in the similar range. However, for OD modes present in the first solvation shell, the correlations persist even at longer waiting times of 5 ps as can be seen from Figure 3. Thus, the distribution plots also support the presence of a stronger temporal correlation of vibrational frequencies of OD modes in the ion solvation shell. The joint probability distribution for water molecules in first solvation shell shows two locally centered distributions. While the distribution centered along the lower frequency can be attributed to the water molecules with strong ion-water hydrogen bonds, the distribution centered at high frequency should refer to the remaining water molecules which are in solvation shell but are having water-water hydrogen bonds.

Finally, we have also calculated the ion-water hydrogen bond and residence time correlations ${ }^{64,65}$ of water in the solvation shell of the fluoride ion and the results are shown in Figure 4. The residence time of water molecules in the ion solvation shell is found to be $25.1 \mathrm{ps}$ which is in reasonably good agreement with the third decay component of FTCF for the first solvation shell. Further, the ion-water hydrogen bond lifetime obtained by integrating the continuous hydrogen bond correlation function is found to be around 5.7 ps.

\subsection{Echo intensity and S3PE}

The echo signal measured in vibrational echo peak shift experiments corresponds to the absolute value of the third order polarization. In Figure 5 (a), we have shown the integrated echo intensity along the rephasing direction as function of different waiting times $t_{2}$. The integrated echo intensity is shown for the bulk water. For the smaller values of $t_{2}$, the intensity shows a peak which is often referred to as the vibrational echo. However as the waiting time increases, the echo intensity gradually decays due to inhomogeneous broadening and shows trends similar to the free induction decay (FID) as seen in the pump probe experiments.

The time dependent decay of the three-pulse photon echo peak shift (3PEPS) is known to capture the frequency correlation both at short and long timescales. However, the extraction of the slower timescales using 3PEPS involves several parameters and numerical fitting. As a result, several analogues have been introduced in the literature which can also extract the frequency correlation decay from the integrated echo intensity like the slope of 3-pulse photon echo (S3PE) as discussed in Section 2. Recent studies have shown that within impulsive limits, S3PE serves comparatively a better metric than the 3PEPS. In our present calculation, we have assumed the applied fields to be delta function pulses, thus the S3PE has been calculated to determine the frequency 


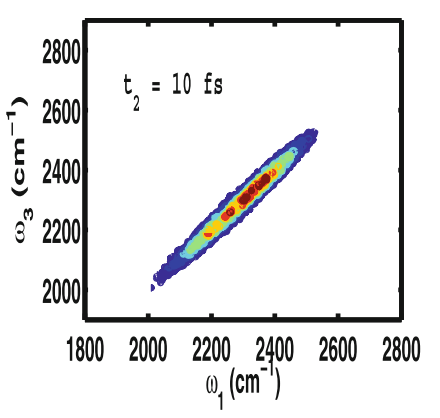

First Solvation Shell
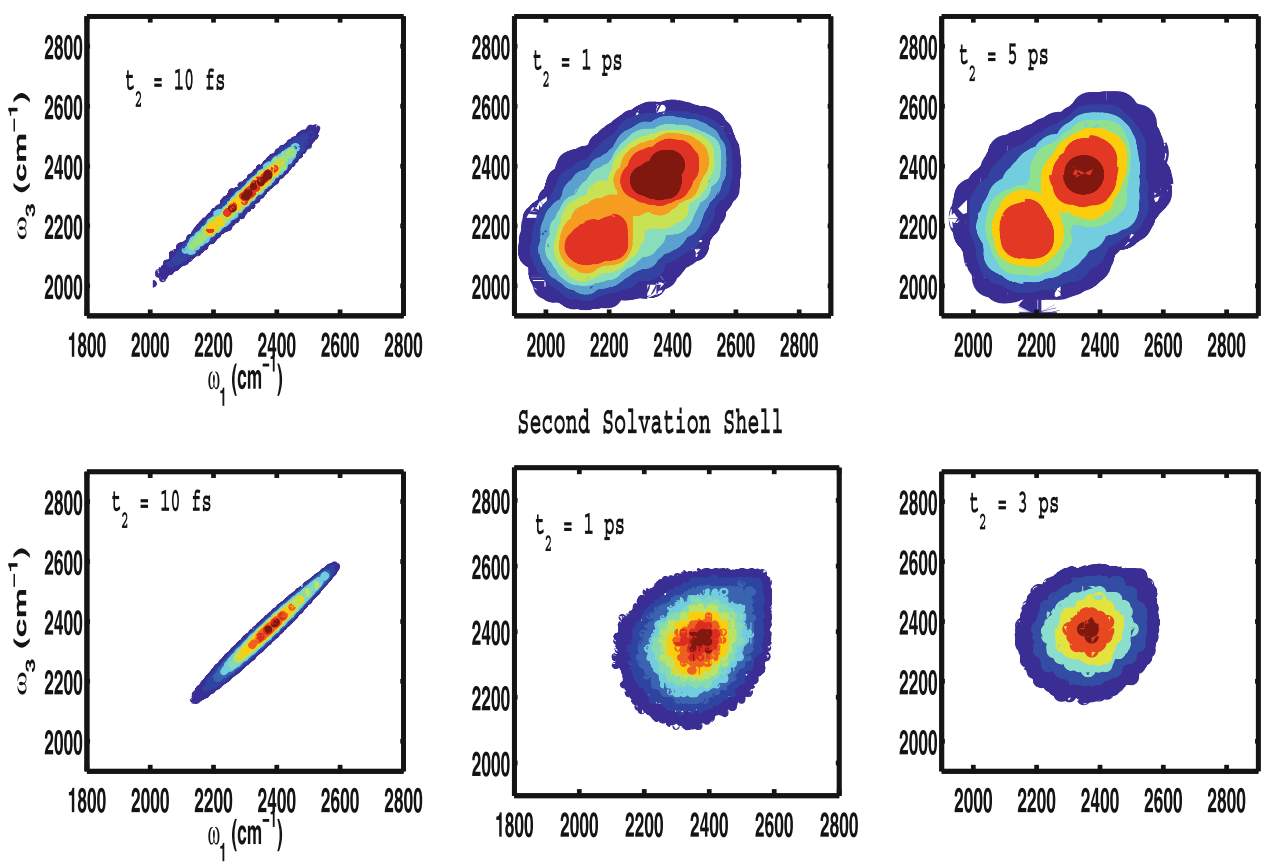

Second Solvation Shell
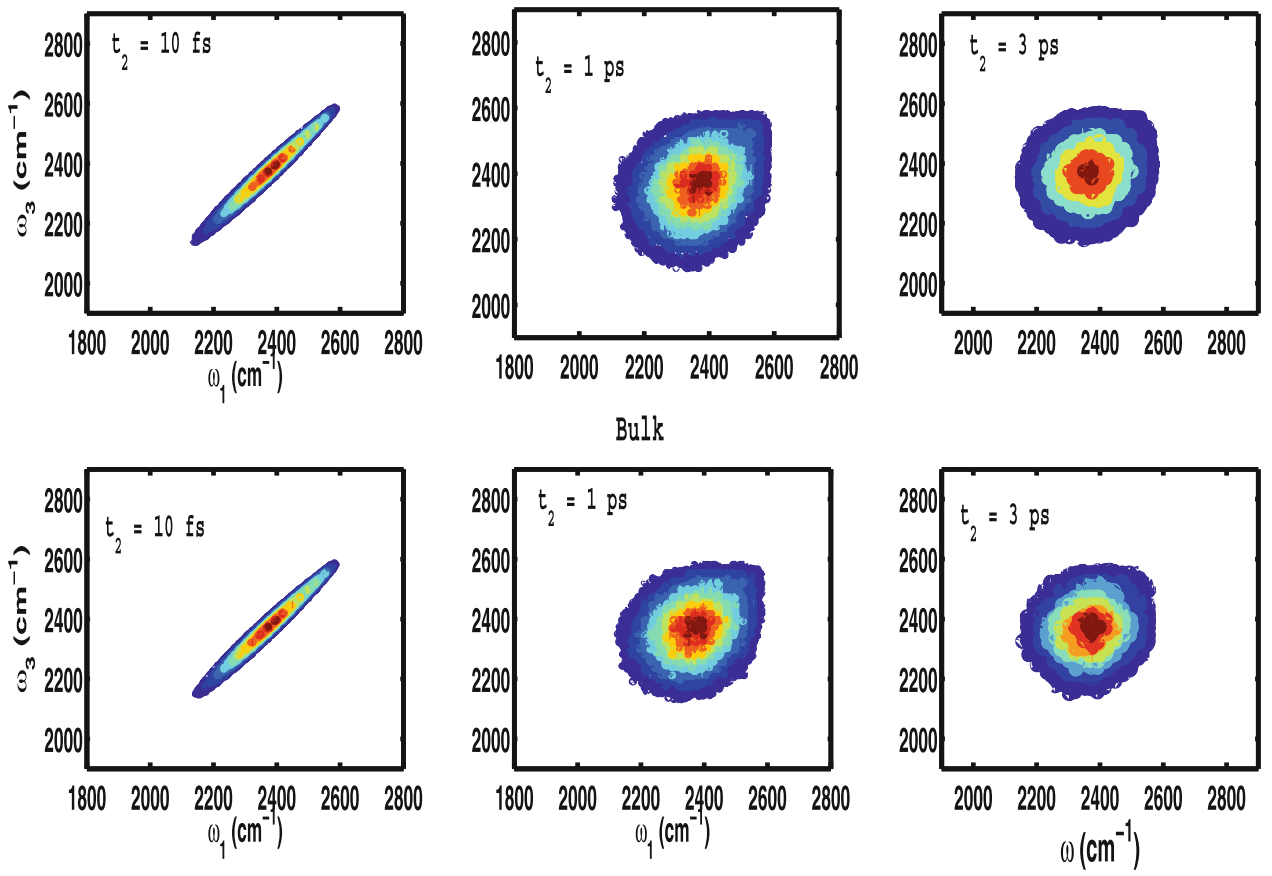

Figure 3. Joint probability distributions of finding frequencies $\omega_{1}$ and $\omega_{3}$ at a time gap of $t_{2}$ for OD modes present in the first and second solvation shells and in the bulk of an aqueous solution containing a fluoride ion.

correlation loss of OD modes in the solvation shells and also in the bulk. The time dependent decay of S3PE for OD modes in the first and second solvation shells and also in the bulk are shown in Figure 5(b). We find that the decay of S3PE for the second solvation shell and the bulk are quite similar at short as well as long timescales. The timescales of spectral diffusion were calculated using a biexponential fit for the second solvation shell and bulk while a triexponential fit was used for the first solvation shell as per Eqs. 14 and 15. For bulk water, the shorter and the longer timescales of decay are found to be $64 \mathrm{fs}$ and $2.48 \mathrm{ps}$, respectively. The longer timescale of decay for OD modes in the second solvation shell is found to be $2.94 \mathrm{ps}$ which is not too different from that of bulk water. For water in the first solvation shell, a fast timescale of $52 \mathrm{fs}$, an intermediate timescale is $8.62 \mathrm{ps}$ and then a longer timescale around $22.4 \mathrm{ps}$ are found. We note that the vibrational dynamics captured by S3PE in the solvation shells and the bulk are found to be in reasonably good agreement with that of FTCF.

\section{$4.3 \quad 2 D-I R$ spectroscopy}

The 2D-IR spectroscopy is known to capture the relaxation of the system in a very short time interval which is comparable to the time scale of vibrational dynamics. Unlike linear absorption spectroscopy which shows absorption averaged over time, 2D-IR spectroscopy captures time dependent absorption which can help to investigate the dynamics of different processes that lead to broadening of the linear spectrum. The 2D-IR spectrum of OD modes oscillating in the first and second solvation shells of the fluoride ion and also in the bulk for the waiting times of $10 \mathrm{fs}, 3 \mathrm{ps}$ and $20 \mathrm{ps}$ are shown in 


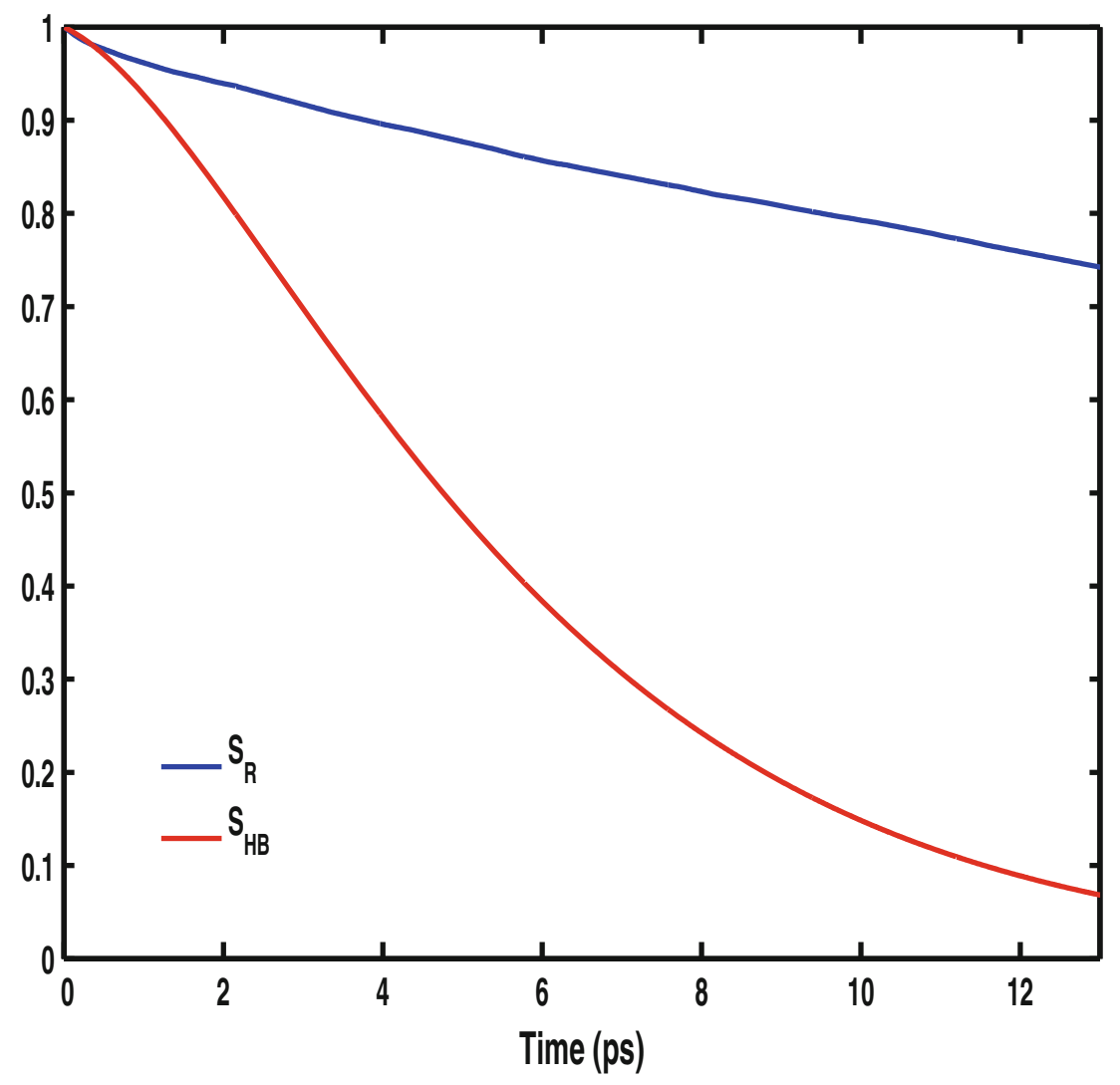

Figure 4. Time dependence of the residence correlation function and continuous ion-water hydrogen bond correlation function of OD modes.
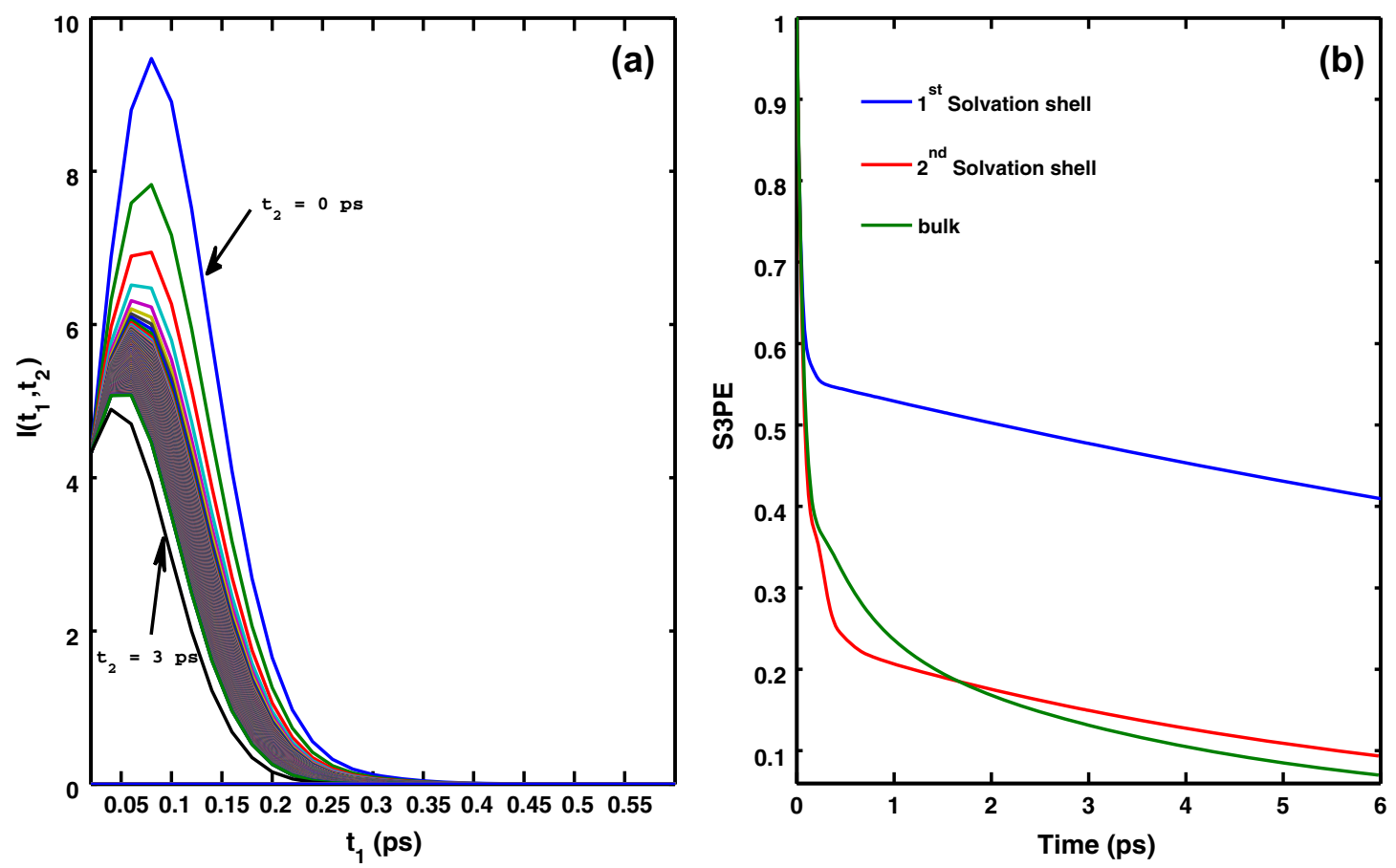

Figure 5. (a) Integrated echo intensity for the bulk water as a function of $t_{1}$, (b) S3PE of the OD stretch modes in the first and second solvation shells of the fluoride ion and in bulk. 


$$
t_{2}=10 \mathrm{fs}
$$
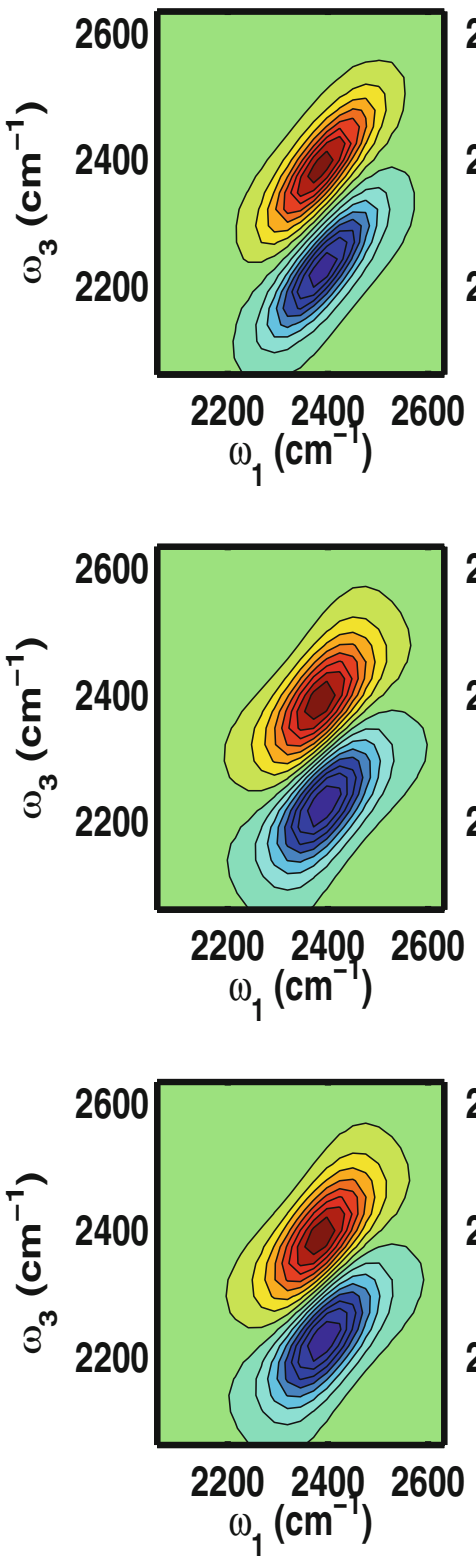

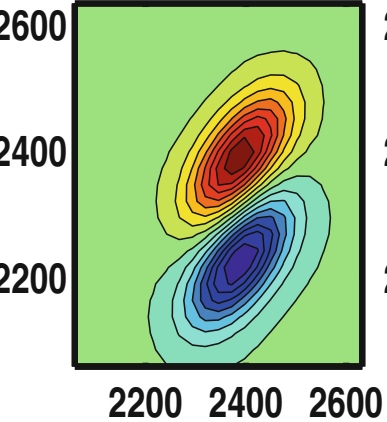

Second Solvation Shell

$t_{2}=3 \mathrm{ps}$ $t_{2}=20$ ps

First Solvation Shell

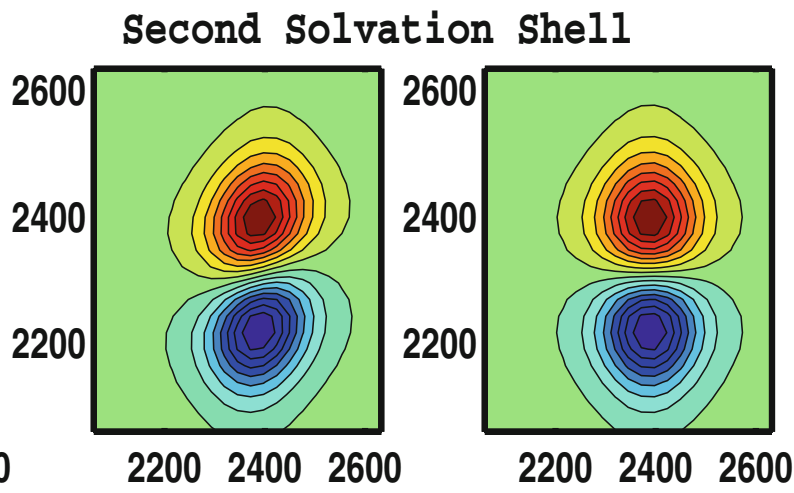

Bulk
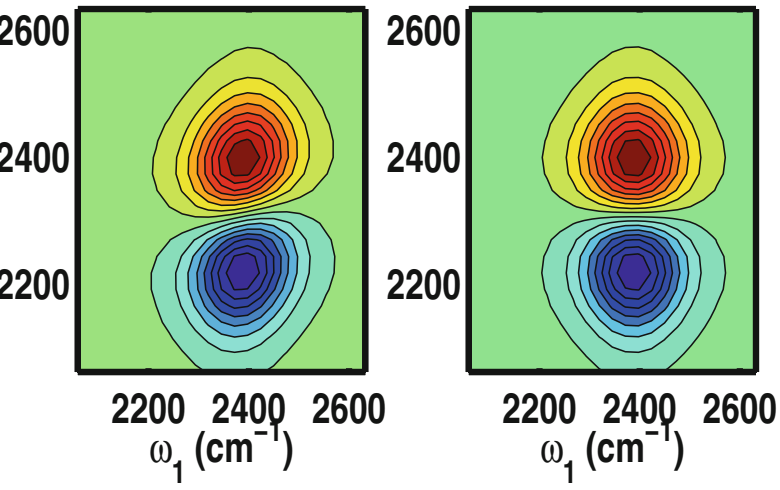

Figure 6. Two dimensional infrared spectra of OD stretch modes in first and second solvation shells of the fluoride ion and in the bulk of an aqueous fluoride solution for different waiting times.

Figure 6. We note that while the nodal line separating the $0 \rightarrow 1$ and $1 \rightarrow 2$ resonances of the spectra for the bulk and second solvation shells get completely aligned with the horizontal frequency $\left(\omega_{1}\right)$ axis by $3 \mathrm{ps}$, the $2 \mathrm{D}$-IR spectrum of the first solvation shell is still asymmetrical and the nodal line is aligned along the antidiagonal even at the waiting time of $20 \mathrm{ps}$. Several metrics have been proposed to obtain the timescales of vibrational spectral diffusion from the 2D-IR spectrum. In the present work, we have used the slope of the nodal line (SNL) to get the timescale of frequency correlation from the 2D-IR spectrum. The time dependent decays of SNL for the 2D-IR spectra of bulk and solvation shells are shown in Figure 7 . The timescales are obtained by using similar fits as already discussed in subsection 4.1. Three timescales are obtained for the first solvation shell which are: A very fast timescale of $30 \mathrm{fs}$ in the sub-picosecond domain, an intermediate timescale of $8.59 \mathrm{ps}$ and a longer timescale of 33 ps. Further, for the second solvation shell, two time scales are found which are $71 \mathrm{fs}$ and $2.94 \mathrm{ps}$. The cor- 


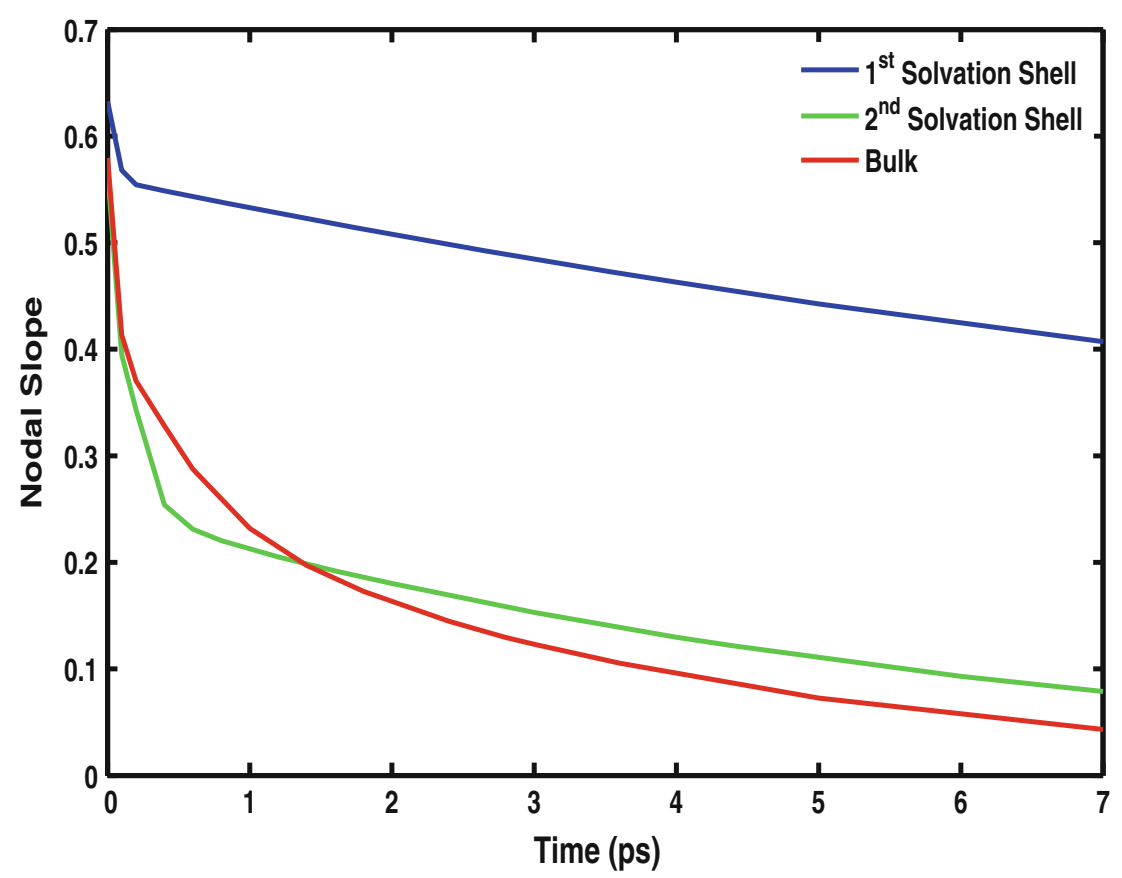

Figure 7. Time dependence of the slope of nodal line (SNL) of 2D-IR spectra of OD stretch modes in the first and second solvation shells of the fluoride ion and in the bulk of an aqueous fluoride solution.

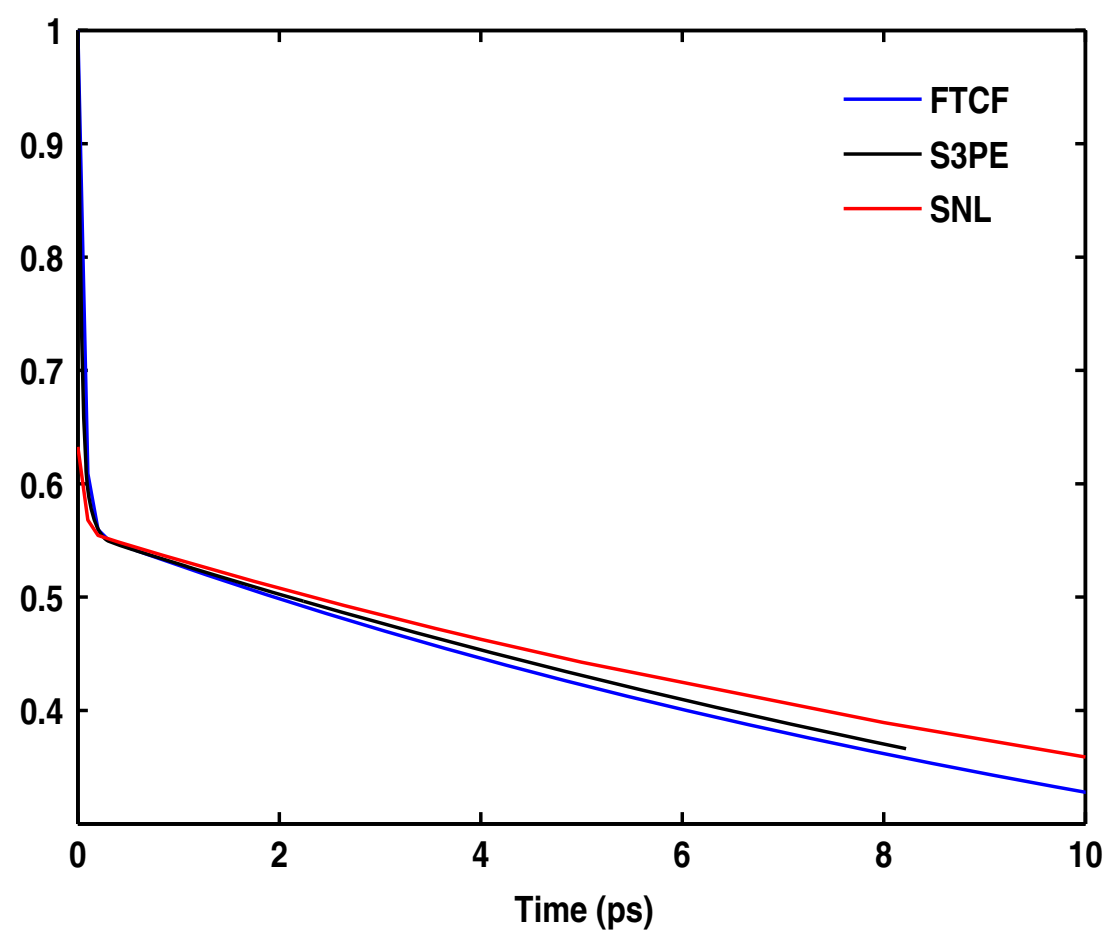

Figure 8. Time dependence of the FTCF (fit), S3PE and SNL of water molecules in the first solvation shell of the fluoride ion in an aqueous fluoride solution.

responding timescales for the bulk solution are found to be $64 \mathrm{fs}$ and 2.48 ps respectively. We note that the timescales for the second solvation shell and the bulk are quite comparable while that of the first solvation shell is slower and has multiple timescales. Further, the timescales obtained for each of the cases are also in good agreement with those found for the decay of S3PE and FTCF. Finally, we have compared the FTCF, S3PE and 
Table 1. Time scales of correlation decay of FTCF and vibrational echo spectral observables for water molecules in the first solvation shell, second solvation shell and the bulk region.

\begin{tabular}{lccc}
\hline Metric & $1^{\text {st }}$ Solv. Shell & $2^{\text {nd }}$ Solv. Shell & Bulk \\
\hline FTCF & $50 \mathrm{fs}, 8.46 \mathrm{ps}, 27.5 \mathrm{ps}$ & $75 \mathrm{fs}, 2.7 \mathrm{ps}$ & $69 \mathrm{fs}, 2.54 \mathrm{ps}$ \\
S3PE & $52 \mathrm{fs}, 8.62 \mathrm{ps}, 22.4 \mathrm{ps}$ & $79 \mathrm{fs}, 2.94 \mathrm{ps}$ & $64 \mathrm{fs}, 2.48 \mathrm{ps}$ \\
SNL & $30 \mathrm{fs}, 8.59 \mathrm{ps}, 33.0 \mathrm{ps}$ & $71 \mathrm{fs}, 2.94 \mathrm{ps}$ & $64 \mathrm{fs}, 2.5 \mathrm{ps}$ \\
\hline
\end{tabular}

SNL as a measure of spectral diffusion timescales in Figure 8. We note that the S3PE and FTCF are in very good agreement both at the short and long timescales. However, the agreement between the SNL and FTCF tends to get even better at longer timescales. In Table 1, we have included all the time scales of decay of FTCF and different metrics of 2D-IR spectrum that are calculated in the current study.

\section{Conclusions}

In the present work, we have performed $a b$ initio molecular dynamics simulation of an aqueous solution containing a fluoride ion. We have investigated the dynamics of water molecules in the solvation shells of the ion by calculating the frequency time correlation function, joint probability distribution and also through calculations of various observables of vibrational echo and 2D-IR spectroscopy like S3PE and SNL. The decay of all the metrics for the second solvation shell and the bulk are found to be very similar at both the short and long timescales. This implies that the effects of the fluoride ion do not extend beyond the first solvation shell. The water molecules in the second solvation shell feel similar local environment as in the bulk. In contrast, the dynamics of water molecules in the first solvation shell is much slower and show multiple timescales with an overall temporal behavior that is different from that of the second solvation shell and bulk regions.

The calculations of residence time, ion-water and water-water hydrogen bond lifetimes show that for water in the bulk and second solvation shell, the longer timescales of decay of the frequency correlations are determined by the water-water hydrogen bond lifetime. For the first solvation shell, two slower timescales are found in the decay of FTCF, S3PE and SNL which are attributed to the ion-water hydrogen bond lifetime and residence time of water in the ion's first solvation shell. Further, we also note that the decay of FTCF is in good agreement with that of S3PE and SNL which is a reaffirmation of the assertion that vibrational echo spectroscopic observables identify the loss of frequency correlation with time.

\section{Acknowledgements}

We gratefully acknowledge the financial support from Science and Engineering Research Board (SERB) through a J.C. Bose Fellowship project to AC and the Council of Scientific and Industrial Research (CSIR), Government of India.

\section{References}

1. Bagchi B 2013 Water in Biological and Chemical Processes: From Structure and Dynamics to Function (UK: Cambridge University Press)

2. Fayer M D 2012 Dynamics of water interacting with interfaces, molecules, and ions Acc. Chem. Res. 453

3. Ohta K, Tayama J, Saito S and Tominaga K 2012 Vibrational frequency fluctuation of ions in aqueous solutions studied by three-pulse infrared photon echo method Acc. Chem. Res. 451982

4. Timmer, R L A and Bakker H J 2009 Hydrogen bond fluctuations of the hydration shell of the bromide anion J. Phys. Chem. A 1136104

5. S Zheng, Zhang W, Ji M, Hartsock R and Gaffney K L 2013 Aqueous Mg2+ and Ca2+ Ligand Exchange Mechanisms Identified with 2DIR Spectroscopy J. Phys. Chem. B 11712268

6. Kropman M F and Bakker H J 2001 Dynamics of water molecules in aqueous solvation shells Science 2912118

7. Omta A W, Kropman M F, Woutersen S and Bakker H J 2003 Negligible effect of ions on the hydrogen-bond structure in liquid water Science 301347

8. Rey R, Møller K B and Hynes J T 2002 Hydrogen bond dynamics in water and ultrafast infrared spectroscopy $J$. Phys. Chem. A 10611993

9. Møller K B, Rey R and Hynes J T Hydrogen bond dynamics in water and ultrafast infrared spectroscopy: a theoretical study J. Phys. Chem. A 1081275

10. Bakker H J and Skinner J L 2009 Vibrational Spectroscopy as a Probe of Structure and Dynamics in Liquid Water Chem. Rev. 1101498

11. Garrett S and Hamm P 2008 Three-Point Frequency Fluctuation Correlation Functions of the $\mathrm{OH}$ Stretch in Liquid Water J. Chem. Phys. 128104507 
12. Ghosh R, Samanta T, Banaerjee S, Biswas R and Bagchi B 2015 Spatio-Temporal Correlations in Aqueous Systems: Computational Studies of Static and Dynamic Heterogeneity by 2D-IR Spectroscopy Faraday Discuss. 177313

13. Biswas R, Furtado J and Bagchi B 2013 Layerwise Decomposition of Water Dynamics in Reverse Micelles: A Simulation Study of Two-Dimensional Infrared Spectrum J. Chem. Phys. 139144906

14. Moilanen D E, Fenn E E, Lin Y S, Skinner J L, Bagchi B and Fayer M D 2008 Water Inertial Reorientation: Hydrogen Bond Strength and the Angular Potential Proc. Nat. Acad. Sci. 1055295

15. Lin Y S, Auer B M and Skinner J L 2009 Water structure, dynamics, and vibrational spectroscopy in sodium bromide solutions J. Chem. Phys. 131144511

16. Soper A K and Weckstrom K 2006 Ion solvation and water structure in potassium halide aqueous solutions Biophys. Chem. 124180

17. Park S and Fayer M D 2007 Hydrogen bond dynamics in aqueous NaBr solutions Proc. Nat. Acad. Sci. 10416731

18. Ohrn A and Karlstrom G 2004 A Combined Quantum Chemical Statistical Mechanical Simulation of the Hydration of $\mathrm{Li}^{+}, \mathrm{Na}^{+}, \mathrm{F}^{-}$, and $\mathrm{Cl}^{-}$J. Phys. Chem. B 1088452

19. Tongraar A and Rode B M 2003 The hydration structures of $\mathrm{F}$ and $\mathrm{Cl}$ investigated by ab initio QM/MM molecular dynamics simulations Phys. Chem. Chem. Phys. 5357

20. Ohtaki H and Radnai T 1993 Structure and dynamics of hydrated ions Chem. Rev. 931157

21. Nigro B, Re S, Laage D, Rey R and Hynes J T 2006 On the Ultrafast Infrared Spectroscopy of Anion Hydration Shell Hydrogen Bond Dynamics J. Phys. Chem. A 110 11273

22. Mallik B S, Semparithi A and Chandra A 2008 A first principles theoretical study of vibrational spectral diffusion and hydrogen bond dynamics in aqueous ionic solutions: $\mathrm{D}_{2} \mathrm{O}$ in hydration shells of $\mathrm{Cl}$ ions J. Chem. Phys. 129194512

23. Ojha D and Chandra A 2015 Ultrafast Vibrational Echo Spectroscopy of Liquid Water from First- Principles Simulations J. Phys. Chem. B 11911215

24. Roy Choudhuri J, Yadav V K, Karmakar A, Mallik B S, and Chandra A 2012 A First-Principles Theoretical study of Hydrogen-bond Dynamics and Vibrational Spectral Diffusion in Aqueous Ionic Solution: Water in the Hydration Shell of a Fluoride Ion Pure Appl. Chem. 8527

25. Jansen T L C, Cringus D and Pshenichnikov M S 2009 Dissimilar Dynamics of Coupled Water Vibrations $J$. Phys. Chem. A 1136260

26. Yeremenko S, Pshenichnikov M S and Wiersma D A 2003 Hydrogen-Bond Dynamics in Water Explored by Heterodyne-Detected Photon Echo Chem. Phys. Lett. 369107

27. Stenger J, Madsen D, Hamm P, Nibbering E T J and Elsaesser T 2002 A Photon Echo Peak Shift Study of Liquid Water J. Phys. Chem. A 1062341

28. Roy S, Pshenichnikov M S and Jansen T L C 2011 Analysis of 2D CS Spectra for Systems with Non-Gaussian Dynamics J. Phys. Chem. B 1155431

29. Hayashi T, Jansen T L C, Zhuang W and Mukamel S 2005 Collective Solvent Coordinates for the Infrared Spectrum of HOD in $\mathrm{D}_{2} \mathrm{O}$ Based on an ab initio Electrostatic Map J. Phys. Chem. A 10964

30. Jansen T L C, Hayashi T, Zhuang W and Mukamel S 2005 Stochastic Liouville Equations for HydrogenBonding Fluctuations and Their Signatures in TwoDimensional Vibrational Spectroscopy of Water $J$. Chem. Phys. 123114504

31. Nibbering E T J and Elsaesser T 2004 Ultrafast Vibrational Dynamics of Hydrogen Bonds in the Condensed Phase Chem. Rev. 1041887

32. Eaves J D, Loparo J J, Fecko C J, Roberts S T and Tokmakoff A and Geissler P L 2005 Hydrogen Bonds in Liquid Water are Broken only Fleetingly Proc. Nat. Acad. Sci. 10213019

33. Fecko C J, Loparo J J, Roberts S T and Tokmakoff A 2005 Local Hydrogen Bonding Dynamics and Collective Reorganization in Water: Ultrafast Infrared Spectroscopy of HOD/D $2 \mathrm{O}$ J. Chem. Phys. 122054506

34. Loparo J J, Roberts S T and Tokmakoff A 2006 Multidimensional Infrared Spectroscopy of Water. I. Vibrational Dynamics in Two-Dimensional IR Line Shapes J. Chem. Phys. 125194521

35. Loparo J J, Roberts S T and Tokmakoff A 2006 Multidimensional Infrared Spectroscopy of Water. II. Hydrogen Bond Switching Dynamics J. Chem. Phys. 125194522

36. Coreclli S A, Lawrence C P, Skinner J L 2004 Combined Electronic Structure/Molecular Dynamics Approach for Ultrafast Infrared Spectroscopy of Dilute HOD in Liquid $\mathrm{H}_{2} \mathrm{O}$ and $\mathrm{D}_{2} \mathrm{O}$ J. Chem. Phys. 1208107

37. Piryatinski A, Lawrence C P and Skinner J L 2003 Vibrational Spectroscopy of HOD in Liquid $\mathrm{D}_{2} \mathrm{O}$. IV. Infrared Two-Pulse Photon Echoes J. Chem. Phys. 1189664

38. Piryatinski A, Lawrence C P and Skinner J L 2003 Vibrational Spectroscopy of HOD in liquid $\mathrm{D}_{2} \mathrm{O}$. V. Infrared Three-Pulse Photon Echoes J. Chem. Phys. 1189672

39. Auer B, Kumar R, Schmidt J R and Skinner J L 2007 Hydrogen Bonding and Raman, IR, and 2D-IR spectroscopy of Dilute HOD in Liquid $\mathrm{D}_{2} \mathrm{O}$ Proc. Nat. Acad. Sci. 10414215

40. Mukamel S 1995 Principles Of Nonlinear Optical Spectroscopy (Oxford-New York: Oxford University Press)

41. Hamm P and Zanni M 2011 Concepts and Methods of $2 D$ Infrared Spectroscopy (New York: Cambridge University Press)

42. Schmidt J R, Corcelli S A and Skinner J L 2005 Pronounced Non-Condon Effects in the Ultrafast Infrared Spectroscopy of Water J. Chem. Phys. 123044513

43. Khalil M, Demirdven N and Tokmakoff A 2003 Coherent 2D IR Spectroscopy: Molecular Structure and Dynamics in Solution J. Phys. Chem. A 1075258

44. Passino S A, Nagasawa Y, Joo T and Fleming G R 1997 Three-Pulse Echo Peak Shift Studies of Polar Solvation Dynamics J. Phys. Chem. A 101725

45. Cho M, Yu J Y, Joo T, Nagasawa Y, Passino S A and Fleming G R 1996 The Integrated Photon Echo and Solvation Dynamics J. Phys. Chem. 10011944

46. Everitt K F, Geva E and Skinner J L 2001 Determining the Solvation Correlation Function from Three-Pulse Photon Echoes in Liquids J. Chem. Phys. 1141326

47. Yang M 2009 Effect of Finite Pulse Duration in Three Pulse Photon Echo Experiments: Numerical Comparison of 3PEPS and S3PE Chem. Phys. Lett. 467304 
48. Car R and Parrinello M 1985 Unified Approach for Molecular Dynamics and Density-Functional Theory Phys. Rev. Lett. 552471

49. Marx D and Hutter J 2009 Ab Initio Molecular Dynamics: Basic Theory and Advanced Methods (Cambridge: Cambridge University Press)

50. Hutter J, Alavi A, Deutsch T, Bernasconi M, Goedecker S, Marx D, Tuckerman M and Parrinello M. CPMD Program, MPI für Festkörperforschung and IBM Zurich Research Laboratory. See www.cpmd.org.

51. Lide D R 2003 CRC Handbook of Chemistry and Physics $84^{\text {th }}$ edn. (Boca Raton: Taylor \& Francis)

52. Kohn W and Sham L J 1965 Self-Consistent Equations Including Exchange and Correlation Effects Phys. Rev. 140 A1133

53. Parr R G and Yang W 1994 Density-Functional Theory of Atoms and Molecules. International Series of Monographs on Chemistry (USA: Oxford University Press)

54. Troullier N and Martins J L 1991 Efficient Pseudopotentials for Plane-Wave Calculations Phys. Rev. B 431993

55. McGrath M J, Kuo I F and Siepmann J I 2011 Liquid structures of Water, Methanol, and Hydrogen Fluoride at Ambient Conditions from First Principles Molecular Dynamics Simulations with a Dispersion Corrected Density Functional Phys. Chem. Chem. Phys. 1319943

56. McGrath M J, Kuo I F, Ghogomu J N, Mundy C J and Siepmann J I 2011 Vapor-Liquid Coexistence Curves for Methanol and Methane Using Dispersion-Corrected Density Functional Theory J. Phys. Chem. B 11511688

57. Yoo S and Xantheas S 2011 The Effect of Dispersion Corrections on the Melting Temperature of Liquid Water J. Chem. Phys. 134121105
58. Lin I C, Seitsonen A P, Coutinho-Neto M D, Tavernelli I and Rothlisberger U 2009 Importance of van der Waals Interactions in Liquid Water J. Phys. Chem. B 1131127

59. Lin I C, Sietsonen A P, Tavernelli I and Rothlisberger U 2012 Structure and Dynamics of Liquid Water from $A b$ Initio Molecular Dynamics - Comparison of BLYP, PBE, and RevPBE Density Functionals with and without van der Waals Corrections J. Chem. Theo. Comput. 83902

60. Grimme S 2004 Accurate Description of van der Waals Complexes by Density Functional Theory Including Empirical Corrections J. Comput. Chem. 251463

61. Grimme S 2006 Semiempirical GGA-Type Density Functional Constructed with a Long-Range Dispersion Correction J. Comput. Chem. 271787

62. Vela-Arevalo L V and Wiggins S 2001 Time-Frequency Analysis of Classical Trajectories of Polyatomic Molecules Int. J. Bifurcation Chaos Appl. Sci. Eng. 11 1359

63. Semparithi A and Keshavamurthy S 2003 Intramolecular Vibrational Energy Redistribution in DCO $\left(\widetilde{X}^{2} A^{\prime}\right)$ : Classical-Quantum Correspondence, Dynamical Assignments of Highly Excited States, and Phase Space Transport Phys. Chem. Chem. Phys. 55051

64. Rapaport D C 1983 Hydrogen Bonds in Water Mol. Phys. 501151

65. Luzar A 2000 Resolving the Hydrogen Bond Dynamics Conundrum J. Chem. Phys. 11310663 